\title{
How Faithful Are N-Body Simulations of Disc Galaxies? - Artificial Suppression of Stellar Dynamical Instabilities
}

\author{
Alessandro B. ROMEO \\ Onsala Space Observatory, Chalmers Univ. of Technology, S-43992 Onsala, Sweden
}

\begin{abstract}
High-softening two-dimensional models, frequently employed in $N$-body experiments, do not provide faithful simulations of real galactic discs. A prescription (\$) is given for choosing meaningful values of the softening length. In addition, a local stability criterion (\$) is given for choosing meaningful input values of the Toomre parameter for a given softening length. Such a criterion should also provide a key to a correct interpretation of computational results in terms of real phenomena.
\end{abstract}

\section{Introduction}

$N$-body simulations employing particle-mesh codes have nowadays become a very powerful tool for investigating the dynamics of disc galaxies. In particular, two-dimensional $N$-body models in which the stars and the cold interstellar gas are treated as two different components have successfully been applied in studies of spiral structure (e.g., Salo 1991; Thomasson 1991). A correct interpretation of computational results in terms of real phenomena poses serious problems, also because there are quantities introduced for numerical reasons which do not have clear physical counterparts. One of such artificial quantities is the softening length of the modified (non-Newtonian) gravitational interaction between the computer particles, and its value can critically affect the results of $N$-body experiments. It is thus of fundamental importance to have a prescription for choosing meaningful values of the softening length. From the stability point of view, it has been suggested that softening introduces a quite reasonable thickness correction for a twodimensional model (e.g., Sellwood 1986, 1987; see also Byrd et al. 1986). In this paper the analogy between numerical softening and finite-thickness effects is investigated in detail on the basis of a local linear stability analysis, and in particular the question "How faithfully does the softening mimic the thickness of galactic discs?" is addressed. It is found that high-softening two-dimensional models, frequently employed in $N$-body experiments, do not provide faithful simulations of real galactic discs. A prescription (\$) is given for choosing meaningful values of the softening length. 
Strictly connected with that problem is the choice of meaningful input values of the local stability parameter for a given softening length. In contrast to the softening length, the Toomre parameter is directly related to observable quantities, has a clear physical meaning, and its output values in $N$-body experiments can be compared to those predicted by theories of spiral structure and secular heating. In this paper a local stability criterion ( $\$$ ) is found in virtue of the descriptive similarity between numerical softening and finite-thickness effects. Such a criterion should indeed provide a key to this problem.

A more thorough discussion is given by Romeo (1993). In this short paper we just focus on a few points.

\section{Local Stability}

It is convenient to adopt the following scaling and parametrization:

$$
\begin{aligned}
& \bar{\lambda} \equiv \frac{k_{\mathrm{H}}}{|k|}, \quad \text { where } \quad k_{\mathrm{H}} \equiv \frac{\kappa^{2}}{2 \pi G \sigma_{\mathrm{H}}} ; \\
& \alpha \equiv \frac{\sigma_{\mathrm{C}}}{\sigma_{\mathrm{H}}}, \quad \beta \equiv \frac{c_{\mathrm{C}}^{2}}{c_{\mathrm{H}}^{2}} ; \quad Q_{\mathrm{H}} \equiv \frac{c_{\mathrm{H}} \kappa}{\pi G \sigma_{\mathrm{H}}} ; \quad \eta \equiv k_{\mathrm{H}} s .
\end{aligned}
$$

In these formulae, $k$ is the local radial wavenumber of the perturbation, $\kappa$ is the epicyclic frequency, $\sigma_{i}$ and $c_{i}(i=\mathrm{H}, \mathrm{C})$ are the unperturbed surface densities and the equivalent planar acoustic speeds of the stars $(H)$ and the cold interstellar gas (C), respectively, $s$ is the softening length of the modified gravitational interaction. The case $\eta=0$ represents the limit of an unsoftened gravitational interaction. There exists a critical value of the softening length beyond which the model is locally stable even for vanishing $Q_{\mathrm{H}}^{2}$ :

$$
\text { Stability of Cold Models : } s>s_{\text {crit }}=\frac{1}{\mathrm{e}} \frac{2 \pi G \sigma}{\kappa^{2}},
$$

$\sigma$ being the total unperturbed surface density. This two-component extension of Miller $(1972,1974)$ criterion for cold models $\left(c_{i}=0\right)$ is indeed the limiting case of a more general local stability criterion for cool models $\left(c_{i}>0\right)$, which can be viewed as the softened two-component extension of Toomre (1964) criterion:

* Stability of Cool Models : $Q_{\mathbf{H}}^{2}>\bar{Q}^{2}$

$\bar{Q}^{2}=\bar{Q}^{2}(\alpha, \beta, \eta)$ being the global maximum of the marginal stability curve derived by Romeo (1993). In particular, in low-softening standard stardominated regimes

$$
\bar{Q}^{2} \approx 1+4(\alpha-\eta) \quad[\alpha \ll 1 ; \beta, \eta=O(\alpha)] .
$$



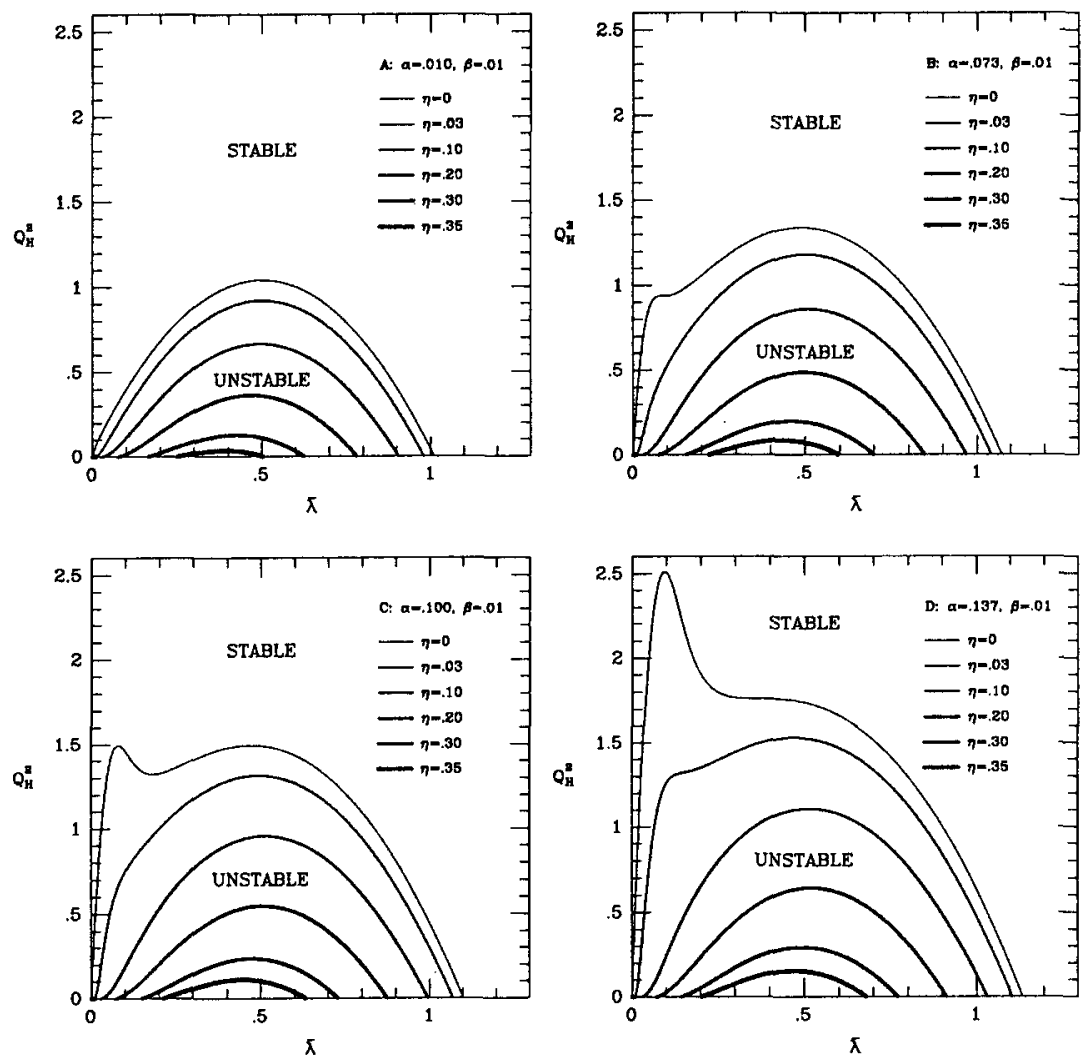

Fig. 1. Two-fluid marginal stability curves in the $\left(\bar{\lambda}, Q_{H}^{2}\right)$ plane for some values of the local parameters $\eta, \alpha$ and fixed $\beta=0.01$. The case $\eta=0$ represents the limit of an unsoftened gravitational interaction

\section{Results}

In presenting the results of the local stability analysis performed in this paper, we have considered the standard star-dominated and the peculiar gasdominated regimes already investigated in the context of thick two-component galactic discs (Romeo 1990, 1992). The marginal stability curves shown in Fig. 1 should qualitatively be compared to those shown in Fig. 4 of Romeo (1992). It is apparent that, because of the highly stabilizing role of numerical softening, the local linear stability properties of two-dimensional $N$-body models can indeed be considerably different from those of thick galactic discs, as derived analytically. In particular, note the suppression of the gaseous peak in peculiar gas-dominated regimes even for exceedingly low softening. The softening can faithfully mimic the thickness of galactic discs or, more precisely, the effective thickness-scale of the stellar component [defined in 
Eq. (6) of Romeo (1992)] only in standard star-dominated regimes, provided the softening length is chosen to be very short compared to the characteristic wavelength corresponding to the stellar peak:

$$
\text { - } s \ll \frac{1}{2} \frac{2 \pi G \sigma_{\mathrm{H}}}{\kappa^{2}} \sim 1 \mathrm{kpc}, \text {. }
$$

as a typical value for realistic $N$-body models of disc galaxies. Softening lengths comparable to the critical value given by the Miller criterion do not fulfil this prescription.

\section{Concluding Remarks}

The suggestion that softening introduces a quite reasonable thickness correction for a two-dimensional model is quantitatively confirmed in low-softening standard star-dominated regimes. A constant softening length would then ideally correspond to a constant scale-height of the stellar component. On the other hand, a realistic simulation of the vertical structure of disc galaxies would in any case require a proper three-dimensional model.

Although the local stability properties of high-softening two-dimensional $N$-body models are considerably different from those of thick galactic discs, the propagation properties of the spiral waves are still expected to be physically plausible in standard star-dominated regimes, provided the softening length is chosen to be shorter than the critical value given by the Miller criterion [cf. the more restrictive prescription $(\uparrow)$ ]. In choosing the input values of the local stability parameter as well as in comparing its output values to those predicted by theories of spiral structure and secular heating, it should then be borne in mind that the stability threshold is not unity (Toomre 1964 criterion for unsoftened one-component models), but the value given by the local stability criterion ( $\bullet$ ) discussed in Sect. 2.

\section{References}

Byrd G.G., Valtonen M.J., Sundelius B., Valtaoja L., 1986, A\&A 166, 75

Miller R.H., 1972, In: Lecar M. (ed.) Proc. IAU Colloq. 10, Gravitational N-Body Problem. Reidel, Dordrecht, p. 213

Miller R.H., 1974, ApJ 190, 539

Romeo A.B., 1990, PhD thesis, SISSA, Trieste, Italy

Romeo A.B., 1992, MNRAS 256, 307

Romeo A.B., 1993, A\&A (submitted)

Salo H., 1991, A\&A 243, 118

Sellwood J.A., 1986, In: Hut P., McMillan S.L.W. (eds.) The Use of Supercomputers in Stellar Dynamics. Springer-Verlag, Berlin, p. 5

Sellwood J.A., 1987, ARA\&A 25, 151

Thomasson M., 1991, PhD thesis, Chalmers Univ. of Technology, Göteborg, Sweden

Toomre A., 1964, ApJ 139, 1217 